\section{Research News}

\section{Malaria marches on}

Two studies show that the malaria parasite is older and tougher than expected. Previous studies had suggested that $P$. falciparum squeezed through an evolutionary bottleneck less than 10,000 years ago, and supported the notion of relatively low genetic variability. But a large analysis of over 204 genes from strains throughout the world now suggest a different scenario: the last common ancestor of all the strains existed between 100,000 and 180,000 years ago, according to $\mathrm{Su}$ and colleagues in the 18 July issue of Nature. The results are bad news for vaccine researchers, who now must contend with a greater parasite diversity than expected. The same research group also sent a warning to public health experts in a second study in Nature analyzing chloroquine resistance. Their analysis suggests that chloroquine resistance has evolved independently at least 4 times, twice in South America, and once each in Papua New Guinea and Southeast Asia. Sequencing of the region containing the chloroquine-resistance gene suggested that complex combinations of mutations arise rapidly, and supports the notion that the parasite could readily develop multidrug resistance. The spread of chloroquine resistance has occurred even more rapidly than previously thought-invading Africa from its Southeast Asian origins in less than 15 years.

\section{Nano-sized destruction}

Researchers have zapped tumors in mice by spraying them with nanoparticles. Hood et al. engineered the particles to hone in on the blood supply that nourishes tumors, according to their report in the 28 June issue of Science. The particles were coated with a molecule that binds $\alpha_{v} \beta_{3}$ integrin, which is expressed mostly on newly growing blood vessels. $\alpha_{v} \beta_{3}$ integrin also serves as a docking site for the internalization of viruses such as the rotavirus, and it mediates the cellular uptake of nanoparticles. The particles carried a payload designed to inactivate the Ras-Raf pathway, a mutant form of Raf cDNA. The Ras-Raf pathway is critical for the formation of new blood vessels, and knocking it out choked off blood vessels and the tumors they nourish. The authors report that a single nanoparticle infusion through an animal's tail could completely shrink massive tumors that were $400 \mathrm{~mm}^{3}$ in size. Whether the technique will show similar cancer-busting qualities in humans remains to be seen.

\title{
Sleep lessons
}

If you want to be the ace in the big game then don't shave off the last two hours of sleep. That's one implication of a study by Walker et al. who showed that a good night's sleep improves learning of motor tasks. In the 3 July issue Neuron, they examined the simple task of finger tapping. Human subjects were asked to repeat the same 5-element sequence of finger tapping as fast as possible for 30 seconds. After a night's sleep, the subjects' fingers moved $20 \%$ faster, without loss of accuracy. The new findings address

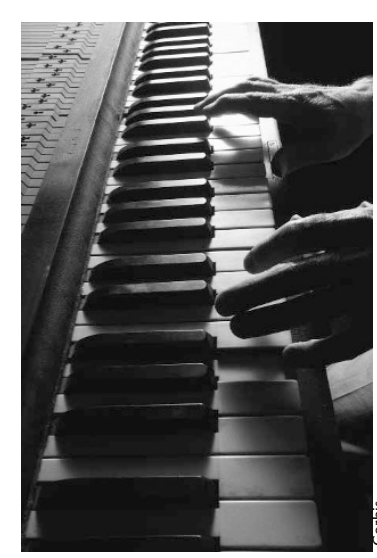

an outstanding controversy of similar studies: whether or not improvement occurs simply because of the passage of time. Subjects trained in the morning showed insignificant improvement after 12 hours of wakefulness. But after a night's sleep, they got the $20 \%$ boost, just like subjects trained in the evening. The findings suggest that a certain type of sleep may account for much of the boost-a type of deep sleep called 2 non-rapid eye movement (2NREM) sleep. About $50 \%$ of our sleeping life is devoted to 2NREM sleep, much of it in the last 2 hours of an eight-hour cycle.

\section{Double-edged gene}

A genetic variant of an immune system gene decreases the risk of atherosclerosis in humans who carry it, but the polymorphism has a flip side. It also increases the risk for bacterial infections, report Kiechl et al. in the 18 July issue of NEJM. The gene encodes the toll-like receptor 4 (TLR4), which responds to lipopolysaccharides on the surface of bacteria. The receptor mediates innate immunity, inducing cytokines, as well as other adhesion and inflammatory molecules in response to bacteria. People with this polymorphism were already known to have an impaired inflammatory response. Now Keichl et al. looked at their medical charts. The authors examined individuals originally recruited from a random population sample in Italy for a long-term population-based study of atherosclerosis. Of the 810 subjects, $10 \%$ had the mutant allele-and a $\sim 5$-fold greater frequency of acute bacterial infection over 5 years compared with individuals with wild-type TLR4. Overall, individuals with the polymorphism had fewer symptoms of atherosclerosis by several criteria. The results are consistent with previous studies showing a link between atherosclerosis and the level of systemic inflammation.

\section{Tolerance and TBP}

The placenta provides an immune shield for the foreign body inside a pregnant woman-the fetus. The placenta secretes immunosuppressive molecules and otherwise counters the mom's immune system at the maternal-placental interface. Hobbs et al. in the 12 July issue of Cell have unlocked a piece of the puzzle at this interface. But they started with an interest in an entirely different subject-evolution of a component of the basal transcription machinery, TATA binding protein (TBP). The authors were intrigued by the fact that the 180amino-acid core of TBP is highly conserved in all eukaryotes, but tetrapod vertebrates sport a large and novel N-terminal domain. In their study, Hobbs et al. replaced the endogenous $T b p$ gene in mice with a version that lacks the sequence encoding this $\mathrm{N}$ terminus. They expected a defect in a trait peculiar and obvious to vertebrates, lungs, for instance. But they hit the placenta, and discovered that most mice died in midgestation with defects in this organ. The TBP-truncated mice survived if the researchers supplied them with wildtype placentas or suppressed the mother's immune system. Genetically obliterating a major histocompatibility complex (MHC) light chain also enabled survival, supporting the notion that the $\mathrm{N}$ terminus may normally transcriptionally-regulate MHC expression. Hobbs et al. speculate that the TBP $\mathrm{N}$ terminus coevolved with the adaptive immune system. But placental mammals may have co-opted the N-terminus for their own needs. Finding out how TBP modulates the immune system could have implications for the mechanisms of rejection and evolution.

\section{Charlotte Schubert}

